\title{
A DNA structure-based bionic wavelet transform and its application to DNA sequence analysis
}

\author{
Fei Chen and Yuan-Ting Zhang \\ Joint Research Center for Biomedical Engineering, Department of Electronic Engineering, The Chinese University of \\ Hong Kong, Shatin, NT, Hong Kong
}

\begin{abstract}
DNA sequence analysis is of great significance for increasing our understanding of genomic functions. An important task facing us is the exploration of hidden structural information stored in the DNA sequence. This paper introduces a DNA structure-based adaptive wavelet transform (WT) - the bionic wavelet transform (BWT) - for DNA sequence analysis. The symbolic DNA sequence can be separated into four channels of indicator sequences. An adaptive symbol-to-number mapping, determined from the structural feature of the DNA sequence, was introduced into WT. It can adjust the weight value of each channel to maximise the useful energy distribution of the whole BWT output. The performance of the proposed BWT was examined by analysing synthetic and real DNA sequences. Results show that BWT performs better than traditional WT in presenting greater energy distribution. This new BWT method should be useful for the detection of the latent structural features in future DNA sequence analysis.
\end{abstract}

Keywords: bionic wavelet transform, energy distribution, DNA sequence, indicator sequence, symbol-to-number mapping

\section{Introduction}

A DNA sequence carries nearly all the genomic information of a living organism. To increase our knowledge of human life, its analysis is of great importance and has drawn extensive attention from a number of disciplines (Moore 2000). To date, scientists have completed the sequencing of DNA sequences from human beings and many other organisms at an astonishing rate. Interpreting the meaning of these DNA sequences is one of the most exciting challenges facing us today. An important topic in current DNA sequence analysis is to reveal its structural features, and to elucidate the relationship between the structural features and their biological functions (Fitch and Sokhansanj 2000).

In spite of the extremely large size of DNA sequences, data transform can be used to study the sequences in a more manageable manner and to facilitate the exploration of the underlying structural features. To achieve this aim, various signal processing methods have been successfully used over the last few years, such as spectral analysis (Veljkovic et al 1985; Benson 1990) and neural networks (Fu 1999). For a detailed introduction to genomic signal processing see Anastassiou (2001) and Zhang et al (2002). Among these methods, time-frequency analysis (TFA) has attracted special attention, since it can present, simultaneously, the structure repetition information and its variance along the sequence. Hence, it is helpful to detect the latent periodicities and distinguish them from the simple random repetitions. Generally speaking, the latent structural periodicities of DNA sequences are directly related to particular genomic functions. For instance, the bases in the coding region of a DNA sequence exhibit a period-3 pattern because of the translation of three bases into an amino acid during protein synthesis. Recently, more periodical patterns within DNA sequences have been discovered, such as 10.5-, 20- and 400base periodicities (Trifonov 1998). The 3-periodicity is even found to be dependent on the size of the DNA sequence (Chechetkin and Turygin 1995).

For time-frequency analysis, energy ${ }^{1}$ distribution is an important factor that affects accurate interpretation of the TFA result. The greater the energy distribution, the better the TFA performance. At present, there are three widely used TFA methods: Wigner-Ville distribution (WVD), shorttime Fourier transform (STFT) and wavelet transform (WT). Each has its individual limitation in terms of energy distribution. WVD suffers from cross-terms caused by

Correspondence: Yuan-Ting Zhang, Joint Research Center for Biomedical Engineering, Department of Electronic Engineering, The Chinese University of Hong Kong, Shatin, NT, Hong Kong; tel +852 2609 8459; fax +852 2603 5558; email ytzhang@ee.cuhk.edu.hk 
interference between independent signal components. Such cross-terms obscure the detection of useful spectral patterns (Bolch and Arce 2002). For STFT, the determination of the optimal window length is a difficulty. The usage of STFT is sometimes limited by its fixed resolution over the timefrequency domain, which will degenerate the energy distribution performance. WT can realise resolution adjustment in a flexible way, so that it has high frequency resolution at low frequencies and high time resolution at high frequencies. However, WT is still restricted by its poor frequency and time resolutions at high and low frequencies, respectively. Another disadvantage of the current TFA methods is that they are independent from the structural properties of the analysed signal. It is apparent that adaptive TFA, matching the signal structure, can more efficiently search the structure pattern of the signal.

In recent years, adaptive time-frequency analysis has been extensively studied in the field of signal processing. The adaptation may arise from the active control mechanisms of biosystems or from the structural feature of the signal. When the control mechanism or the structural feature is incorporated, signal processing methods can optimise their performances to obtain desired results. For instance, the cochlear control mechanism in speech analysis has been introduced into wavelet transform, resulting in an active auditory model-based bionic wavelet transform (BWT) that can realise a more flexible 2-D resolution adjustment (Yao and Zhang 2001). The potential applications of this novel adaptive WT in processing speech signals for cochlear implants were also described by Yao and Zhang (2002). The aim of this paper is to introduce a DNA structure-based bionic wavelet transform to achieve greater energy distribution in DNA sequence analysis.

\section{Symbol-to-number mapping in DNA sequence analysis}

A DNA sequence is linked by a string of nucleotides, or bases. There are four types of nucleotides: adenine (A), thymine $(T)$, guanine $(\mathrm{G})$ and cytosine $(\mathrm{C})$. DNA sequences are described in the symbolic form by using the set of letters $\{\mathrm{A}, \mathrm{T}, \mathrm{G}, \mathrm{C}\}$, eg '...AGTCTGA...'. However, current digital signal processing techniques deal with numerical sequences rather than symbolic strings. This difference has been the major reason that the signal processing field does not have significant impact in genomic sequence processing (Anastassiou 2001). Therefore, for DNA sequence processing, the first step is to transfer the symbolic DNA sequence into a numerical sequence. Generally, the transferred numerical sequence is treated as a discrete-time signal with a sampling rate of $1 \mathrm{~Hz}$. Several mapping methods have been proposed in the literature. In Anastassiou (2001), the conjugate complex numbers $1+i, 1-i,-1+i$ and -1 -i were assigned to the bases A, T, G and C, respectively. This mapping can reflect the complementary structure of DNA double strands. Cosic (1994) used a magnetic energy transmission model for the determination of mapping values. Based on the potential of electron-ion interaction (PEII), the mapping values were calculated to be $\mathrm{A}=0.1260$, $\mathrm{T}=0.1335, \mathrm{G}=0.0806$ and $\mathrm{C}=0.1340$. Voss (1992) introduced a statistical 'walk'-based mapping and used 1 and -1 to represent the occurrence of purine and pyrimidine. The 'walk' curve reflected the trend of the occurrence of purine and pyrimidine. This mapping has been widely used to statistically study the correlation feature of the DNA sequence (Voss 1992). In a similar way, Berger et al (2002) utilised a complex-number-based 3-D 'walk' mapping, with

symbolic sequence $x_{n}$ indicator vectors $y_{n}$ numerical sequence $z_{n}$

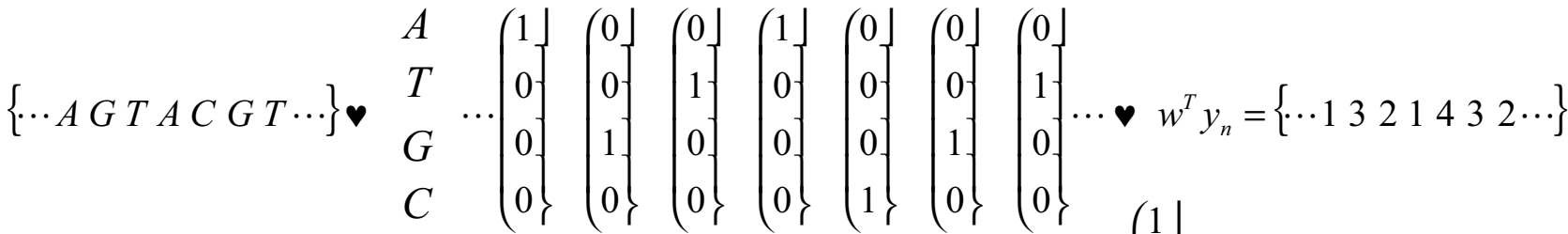

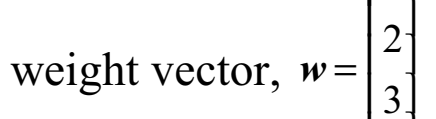

$$
\begin{aligned}
& \text { « } 41 / 4
\end{aligned}
$$

Figure I Mapping process from symbolic sequence $x_{n}$ to numerical sequence $z_{n}$. The indicator vector $\boldsymbol{y}_{n}$ represents the occurrence of the DNA base at index $n$. From top to bottom, four rows in the indicator vector represent the four DNA bases A, T, G and C, respectively. Therefore, a DNA sequence can be separated into four channels of sequences. Weight vector is used to map the indicator vectors to a numerical sequence by the inner product operation. Here, the weight vector is exemplified by $\boldsymbol{w}=[1,2,3,4]^{\top}$ (Wang and Johnson 2002). 


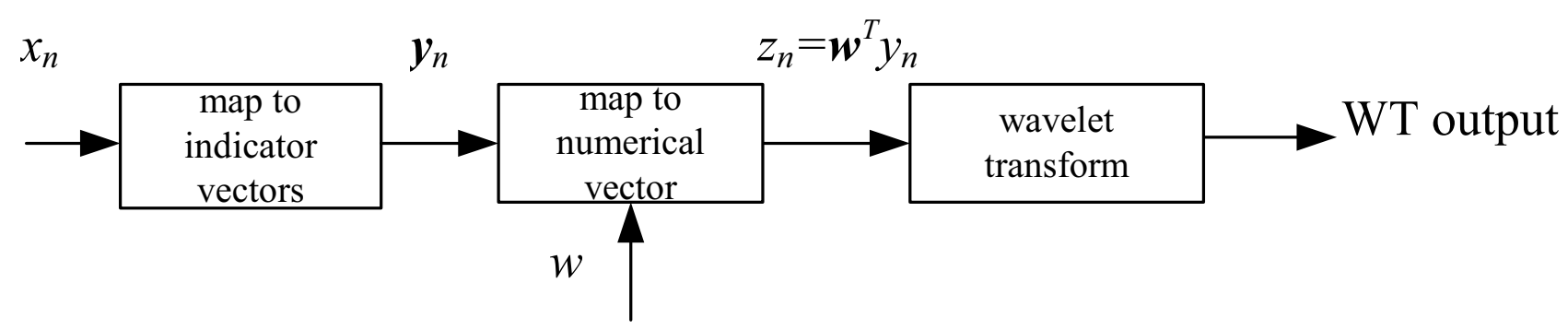

Figure 2 WT analysis of symbolic sequence based on fixed symbol-to-number mapping.

$\mathrm{A}=1, \mathrm{G}=-1, \mathrm{~T}=\mathrm{i}$ and $\mathrm{C}=-\mathrm{i}$, which easily distinguished the purine and pyrimidine by real and complex numbers.

Obviously, the mapping from symbol to number will impose a new mathematical structure that does not exist in the original DNA sequence. For instance, the mapping of $\mathrm{A}=2$ and $\mathrm{T}=1$ assumes that base $\mathrm{A}$ is larger than base $\mathrm{T}$. However, such numerical ordering is meaningless to the nucleotides. Most of the current mapping methods are inherently fixed in that they use invariant values to transfer the DNA bases, regardless of the specific structure of the DNA sequence. It has been agreed that the selection of the numerical mapping should be problem specific, since different mappings may highlight different structural properties (Bolch and Arce 2002). Therefore, using sequence-dependent and adaptive mapping is more desirable. Wang and Johnson (2002) reported an adaptive mapping method, which was based on the criterion to maximise the energy distribution of the output from linear transform. However, such adaptive mapping was not incorporated into the transform. In the following section, we introduce this adaptive mapping into WT, and realise an adaptive WT - ie a DNA structure-based BWT - for DNA sequence analysis.

\section{Methodology}

For mathematical simplicity, before obtaining the numerical sequence, the DNA sequence is first mapped to a set of indicator vectors $\boldsymbol{y}_{n}$, where $n$ is the base index along the DNA sequence. The indicator vector is used to tell which base occurs at index $n$. From top to bottom, four rows of the indicator vector represent four DNA bases of A, T, G and $\mathrm{C}$, respectively. The row of the indicator vector $\boldsymbol{y}_{n}$ will have a ' 1 ' when its corresponding DNA base occurs at index $n$, otherwise a ' 0 '. The mapping values are represented by a vector $\boldsymbol{w}$ (weight vector), as shown in Figure 1. The weight vector has the same dimension as the indicator vector and determines the mapping value of the DNA base at the same row. The resultant mapped numerical sequence $z_{n}$ is obtained by the inner product operation of the weight vector and the indicator vector; that is, $z_{n}=\boldsymbol{w}^{\mathrm{T}} \boldsymbol{y}_{n}$, where $\mathrm{T}$ denotes vector transposing. Consequently, wavelet transform is used to decompose the numerical sequence by:

$$
\mathrm{WT}(n, f)=\mathrm{WT}\left(z_{n}\right)=\mathrm{WT}\left(\boldsymbol{w}^{\mathrm{T}} \boldsymbol{y}_{n}\right)
$$

where $f$ is the analysis frequency of WT. Since the mapping operation is a linear transform at each time-shift $n$ (Wang and Johnson 2002), the WT of the numerical DNA sequence can be reorganised as:

$$
\mathrm{WT}(n, f)=\boldsymbol{w}^{\mathrm{T}} \mathrm{WT}\left(\boldsymbol{y}_{n}\right)=\boldsymbol{w}^{\mathrm{T}} \boldsymbol{y}(n, f)
$$

where $\boldsymbol{y}(n, f)=\mathrm{WT}\left(\boldsymbol{y}_{n}\right)$ is the WT of the indicator matrix consisting of indicator vectors. In other words, each row of the indicator vectors will construct a new sequence, called an indicator sequence. Hence, a DNA sequence can be separated into four channels of indicator sequences (see Figure 1). Consequently, the WT for a DNA sequence becomes a 4-channel transform, with the transform of each indicator sequence separately.

Based on the mapping property, two types of mapping can be used in WT: fixed mapping and adaptive mapping, shown in Figures 2 and 3, respectively. The determination of the adaptive mapping, or weight vector, is critical as indicated above. It can be realised by an optimisation criterion to maximally highlight the WT energy output of the signal in the time-frequency domain. This optimisation is equivalent to maximising the squared magnitude of the WT coefficients:

$$
\begin{aligned}
\left|\boldsymbol{w}^{\mathrm{T}} \boldsymbol{y}(n, f)\right|^{2} & =\left\langle\boldsymbol{w}^{\mathrm{T}} \boldsymbol{y}(n, f), \boldsymbol{w} \boldsymbol{y}(n, f)^{\prime}\right\rangle \\
& =\boldsymbol{w}^{\mathrm{T}}\left\langle\boldsymbol{y}(n, f), \boldsymbol{y}(n, f)^{\prime}\right\rangle \boldsymbol{w}
\end{aligned}
$$

where ' denotes conjugate transposing.

Given the normalisation constriction $\|\boldsymbol{w}\|^{2}=1$, the above energy optimisation corresponds to maximise the Rayleigh quotient of $\left(\boldsymbol{w}^{\mathrm{T}}\left\langle\boldsymbol{y}(n, f), \boldsymbol{y}(n, f)^{\prime}\right\rangle \boldsymbol{w}\right) /\left(\boldsymbol{w}^{\mathrm{T}} \boldsymbol{w}\right)$. Such maximisation is realised when $\boldsymbol{w}$ is proportional to the eigenvector corresponding to the largest eigenvalue of $\left\langle\boldsymbol{y}(n, f), \boldsymbol{y}(n, f)^{\prime}\right\rangle$ (Johnson and Dudgeon 1993). Based on 


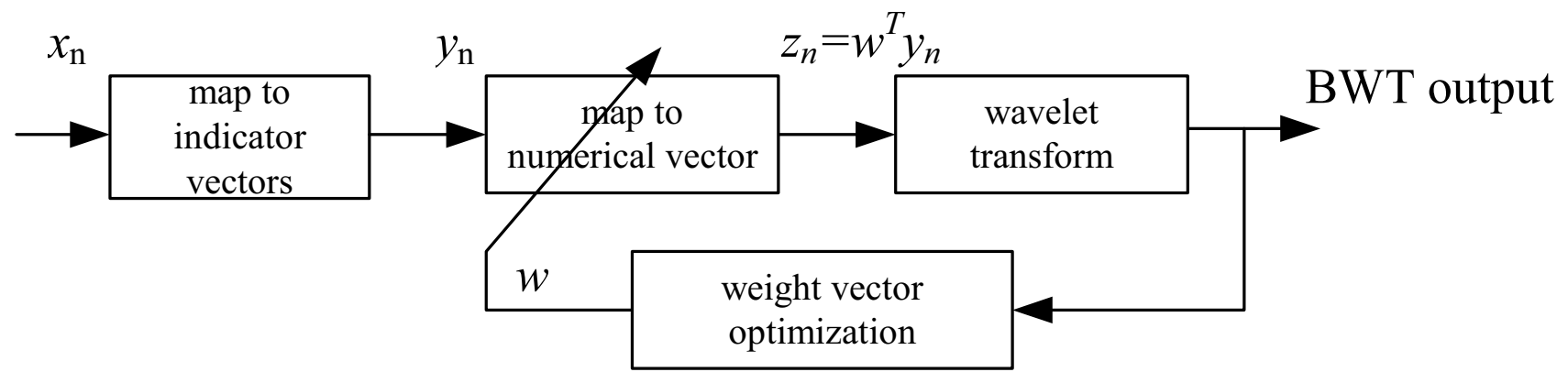

Figure 3 BWT analysis of symbolic sequence based on adaptive symbol-to-number mapping. The optimisation can maximise the BWT energy output and adjust the weight vector. Thus, the whole BWT calculation is adaptive and nonlinear.

this optimisation criterion, the weight vector can be adjusted adaptively at each time-shift $n$ and frequency $f$ in WT. Such optimisation is dependent on the structural feature of the symbolic sequence. Therefore, the whole WT is also realised in an adaptive and nonlinear way to achieve greater energy distribution. We give the name BWT to this DNA structurebased adaptive WT.

\section{Results}

To show the performance of the proposed BWT, a synthetic DNA sequence was analysed, as shown in Figure 4. The synthetic sequence is a string of randomly generated DNA bases interspersed by a periodical region at the middle region. The periodical pattern is a period of 4 or a frequency of $0.25 \mathrm{~Hz}$, shown as 'AATTAATT ...'. Figures 5 and 6 give the analyses of WT with fixed mapping and BWT with adaptive mapping, respectively. The arbitrary vector $\boldsymbol{w}=[1,2,3,4]^{\mathrm{T}}$ was set as the weight vector in the fixed mapping as well as the initial arbitrary weight vector in the adaptive mapping. In Figure 5, the energy of the useful periodical pattern is presented with lower amplitudes than that of the random bases at the left and right regions. Therefore, it is very difficult to detect the periodical pattern at the middle of the WT output. By contrast, in Figure 6, the periodical pattern could still be detected around the frequency of $0.25 \mathrm{~Hz}$ at the middle region. Hence, the existence of the periodical structure is highlighted in the BWT output. Such highlighting is realised by the adaptive adjustment of the weight vectors. Figures 7 and 8 display the mapping values for bases $\mathrm{A}$ and $\mathrm{G}$ along the sequence at the frequency of $0.25 \mathrm{~Hz}$. In the periodical region, the mapping values are adjusted to intensify the energy output. Since base A participates in the periodical pattern, its mapping value is increased. Conversely, the mapping value for base $\mathrm{G}$ is suppressed, since it is not involved in the periodical region.
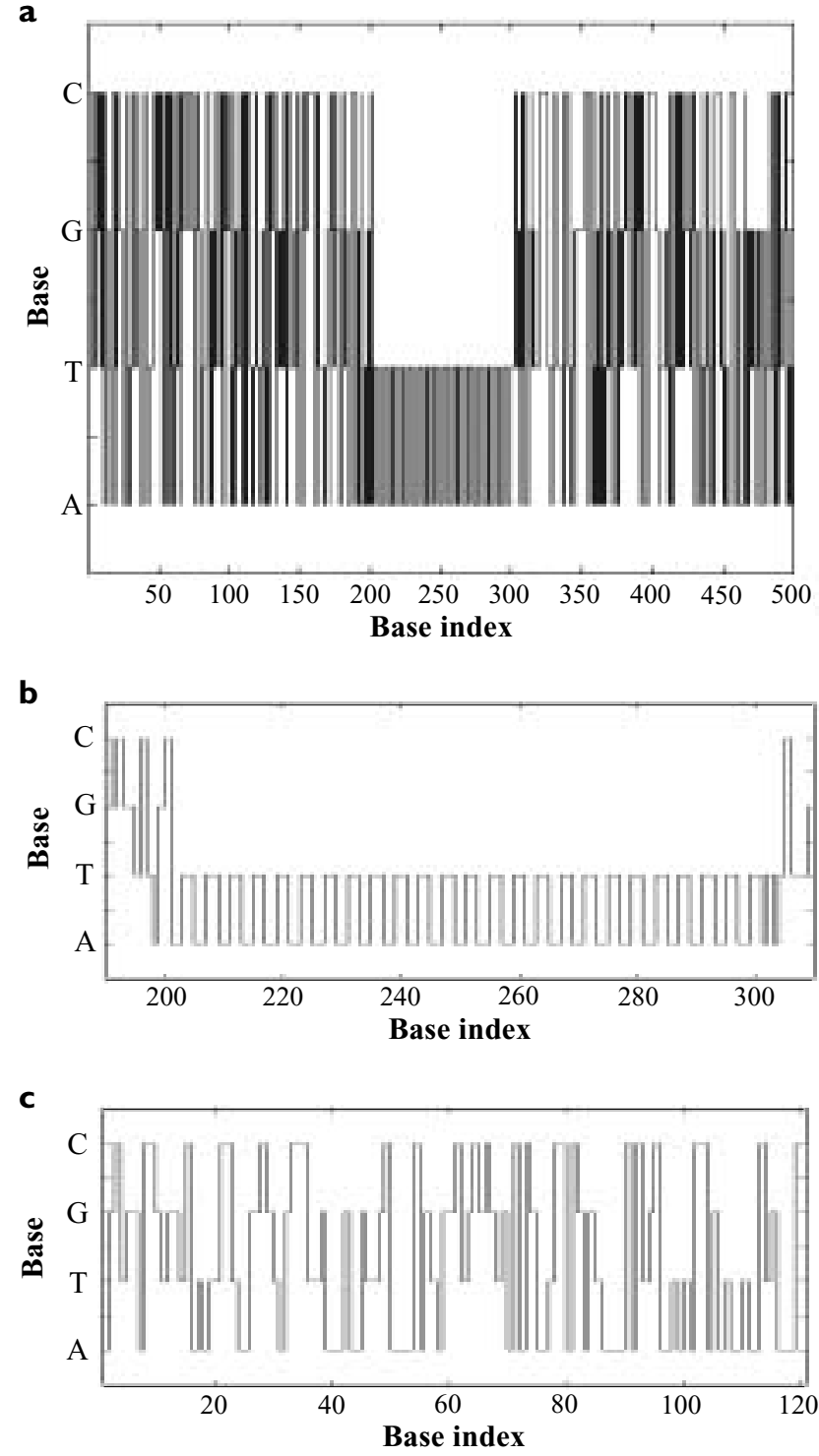

Figure 4 (a) A synthetic DNA sequence with a length of 500 . (b) A periodical structure with a period-4 pattern of 'AATTAATT...' lies at the middle region from base index 201 to 300 . Left and right regions are with randomly generated bases. (c) Part of the left region. 


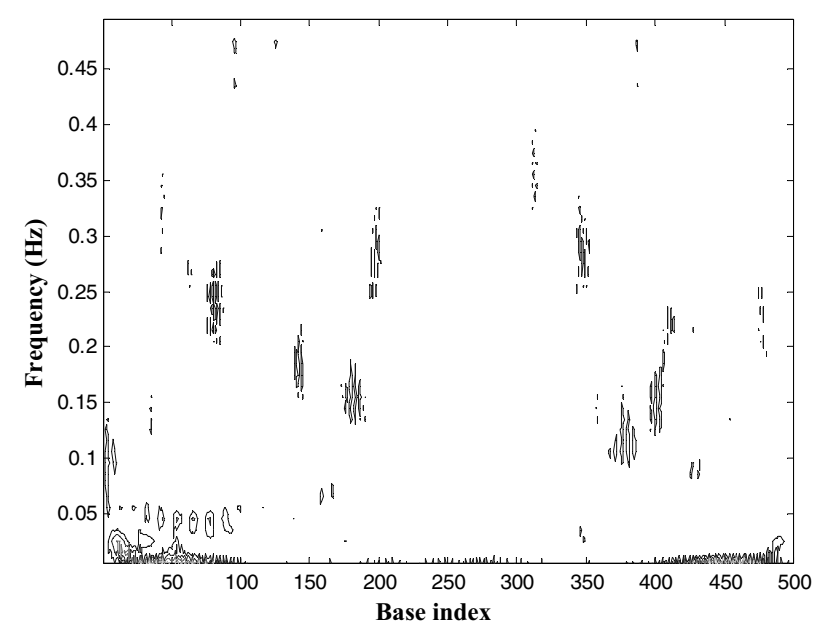

Figure 5 WT analysis of the synthetic DNA sequence based on fixed mapping.

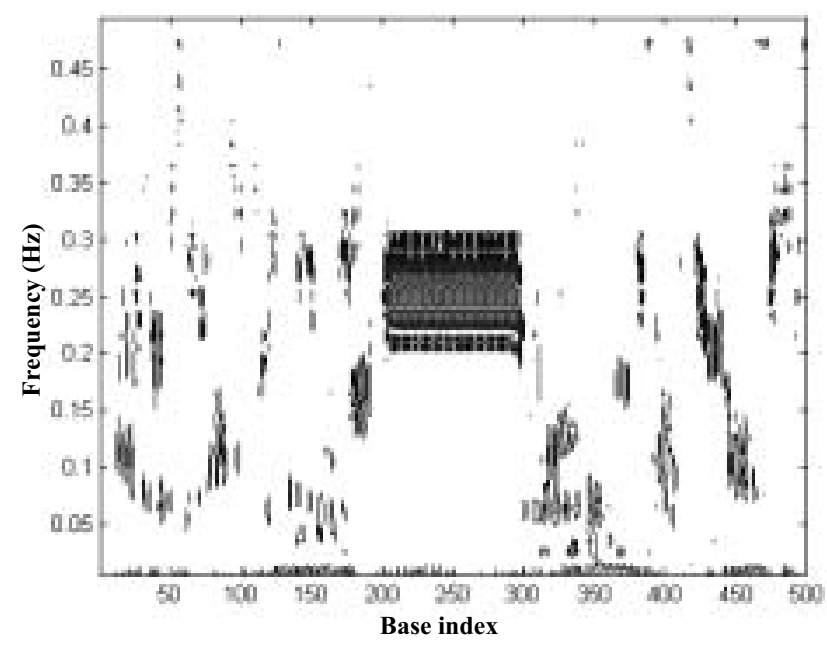

Figure 6 BWT analysis of the synthetic DNA sequence based on adaptive mapping.

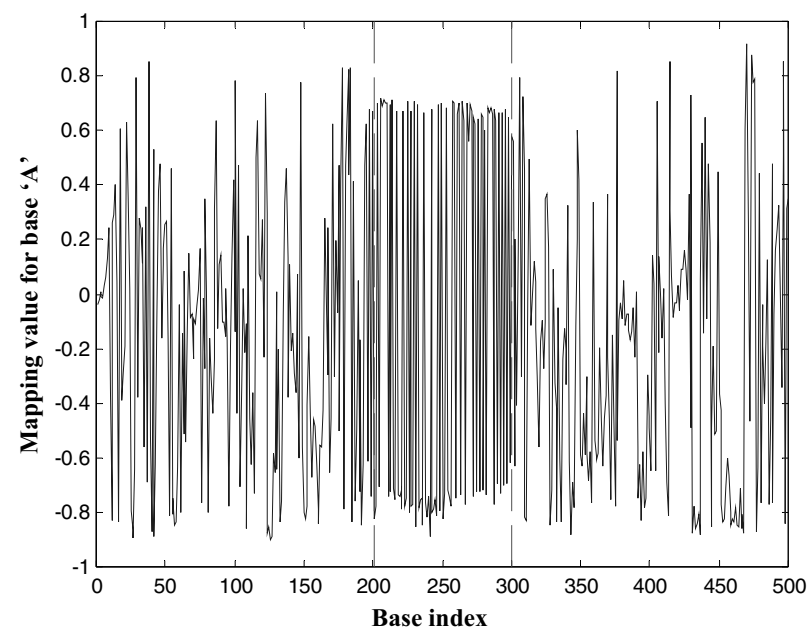

Figure 7 Mapping value for the repetitive base ' $A$ ' at the frequency of $0.25 \mathrm{~Hz}$.

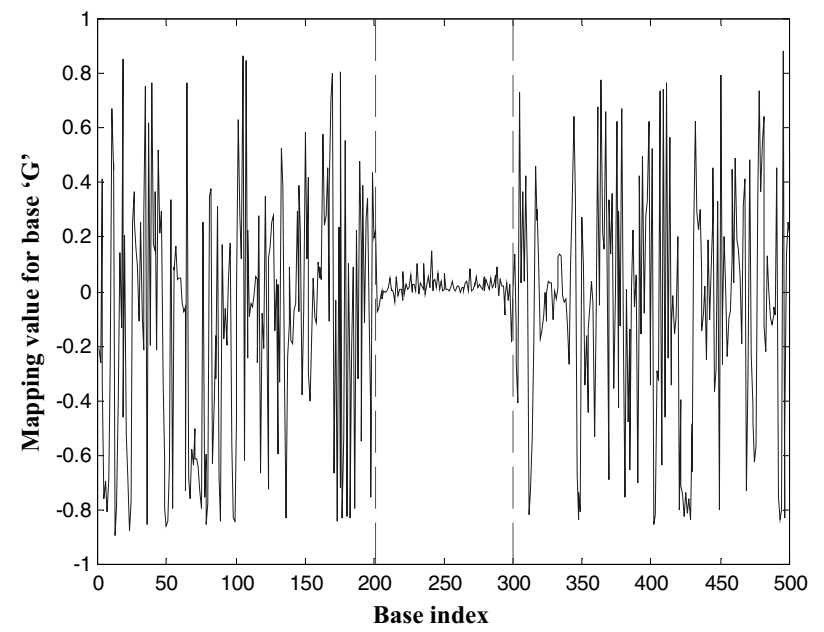

Figure 8 Mapping value for the non-repetitive base ' $G$ ' at the frequency of $0.25 \mathrm{~Hz}$.

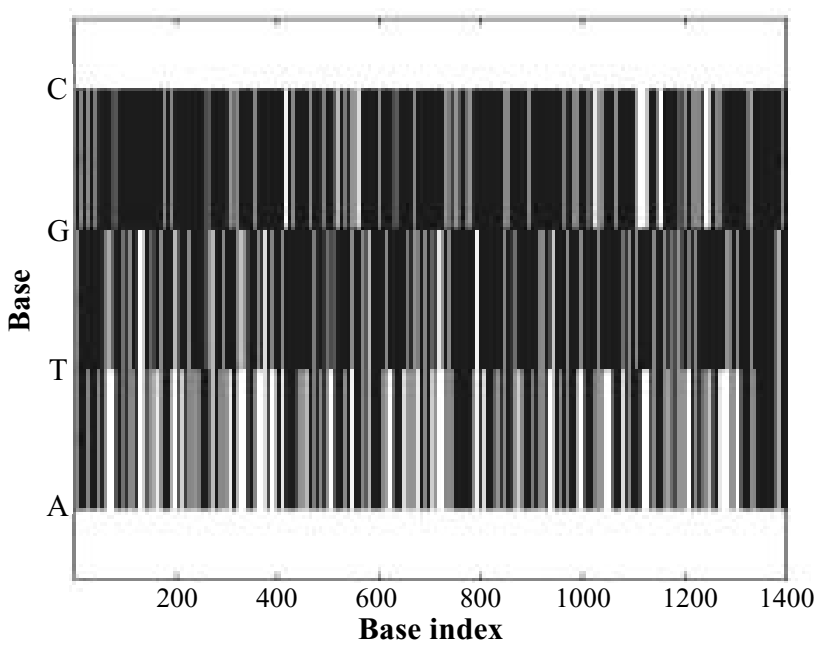

Figure 9 A section of gene sequence from GenBank (accession nr AF440524).

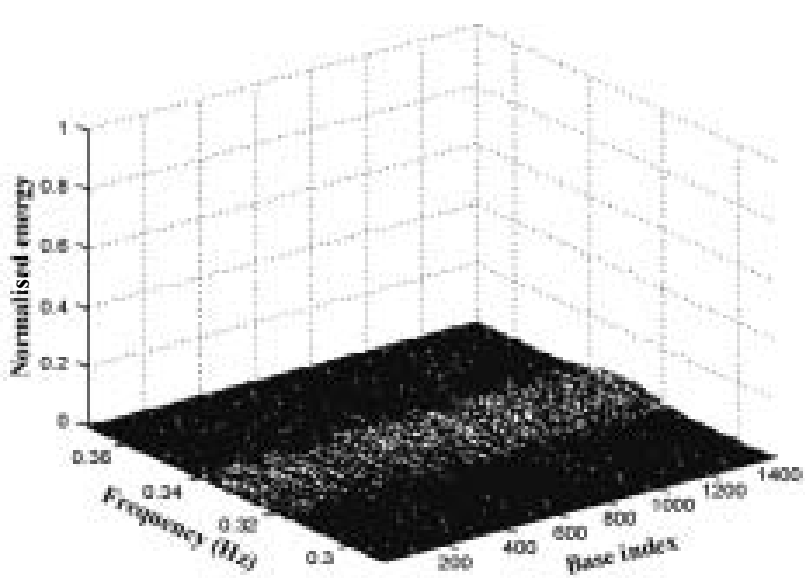

Figure 10 WT analysis of the gene sequence with fixed mapping. 


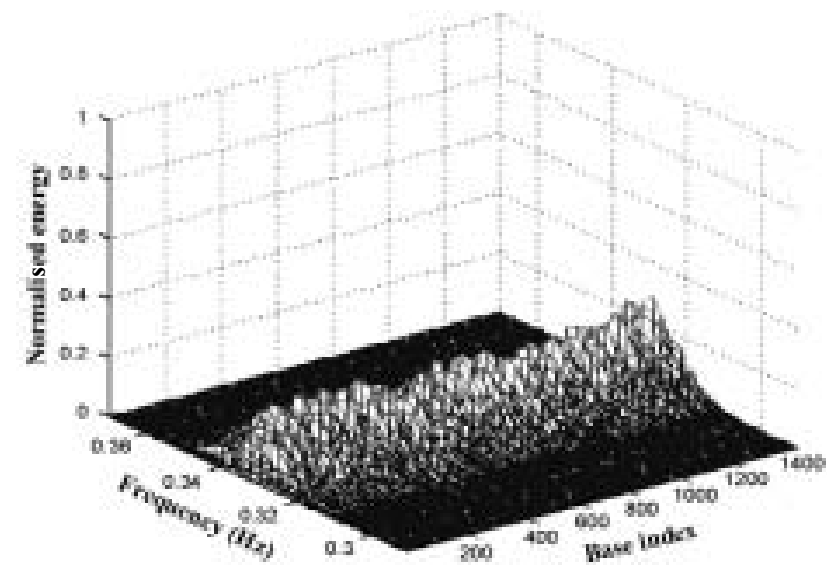

Figure II BWT analysis of the gene sequence with adaptive mapping.

The BWT was also applied to analyse a section of a gene sequence, shown in Figure 9. The gene sequence is accessed from the DNA sequence database GenBank ${ }^{\circledR}$ with accession number of AF440524. Figures 10 and 11 demonstrate the normalised energy outputs from WT and BWT, respectively. To trace the repetition frequency of $0.33 \mathrm{~Hz}$ originating from the gene structure, the frequency range is displayed from 0.3 to $0.36 \mathrm{~Hz}$. BWT gives more concentrated energy distribution around the frequency of $0.33 \mathrm{~Hz}$. By contrast, WT only shows sparse coefficients with low amplitudes around the frequency of $0.33 \mathrm{~Hz}$. The preset weight vector $\boldsymbol{w}$ is the same as that used in the experiment of the synthetic sequence analysis. All the procedures were implemented in the MATLAB ${ }^{\circledR}$ environment.

\section{Discussion}

Real-world information can generally be categorised into two types: symbol and number, exemplified by language and real-valued time signal, respectively. There is a fundamental difference between these two types of signals. The numerical signal satisfies the properties of a mathematical field and has its own mathematical structure. Within a mathematical field, many algebraic operations have been defined to reflect the interrelation between numerical signals. Modern signal processing is based on the mathematical structure of the signal and the operations of the field. Conversely, the symbolic signal is made up from a set of symbols. The symbol set usually does not construct a mathematical field, so that the symbolic signal does not possess the well known mathematical structure. Arithmetic operations are also worthless to the symbols. For instance, we could not compare the difference between bases A and T. Hence, symbol-to-number mapping becomes a necessary and important step for DNA sequence analysis. However, the usage of the mapping should avoid imposing an arbitrary mathematical structure to the symbol sequence, such as Voss's 'walk'-based mapping (Voss 1992). It uses numbers 1 and -1 to merely represent the occurrence of the DNA bases and does not assign an exact value to each base. The adaptive mapping in this paper does not emphasise the mathematical structure of the DNA sequence. It is aimed at maximising the energy distribution of the WT output based on the specific structure of the DNA sequence.

In nature, a DNA sequence is represented by a set of four symbols. Thus, it can be treated as being comprised by four channels of indicator sequences. This 4-channel structure of the DNA sequence provides a way to introduce the energy output maximisation mechanism in timefrequency domain. The WT analysis of each channel will make up the whole WT output by its weight value. In the fixed mapping-based WT, the detected structural pattern of each channel is presented with invariant weight value in the whole WT output. However, in the adaptive mappingbased WT, the weight value could be adaptively modified according to the structure repetition information of the sequence, shown as the optimisation processing in Figure 3. After the optimisation, the weight value of the repetitive base is increased, while that of the non-repetitive base is suppressed. Such weight vector adaptation will optimise WT energy output so that the existence of the underlying periodical structure is highlighted. The adaptive weight vector is determined by the specific structure of the DNA sequence. Hence, the whole WT analysis also becomes data dependent, and an adaptive WT, namely BWT, is then proposed.

Besides optimising the BWT energy distribution, the adaptive mapping is also possible to introduce the interaction among DNA bases. When the fixed numerical mapping is used to transfer the DNA bases into digital numbers, it assumes that the four channels of indicator sequences are independent of each other. However, genomic sequences, including DNA sequences, are generated by a complicated physiological process. There is interaction between adjacent genomic bases, with evidences from the reported long-range correlation in a DNA sequence (Arneodo et al 1995; Herzel et al 1998). Therefore, it does not seem appropriate to analyse the genomic sequence in an independent way. The interaction among bases should be taken into account. Moreover, the base interaction is found to have its particular meanings. For instance, it has been shown that the PEII is correlated with some biological properties of organic molecules, such as toxicity and antibiotic properties. 
(Veljkovic et al 1985). Nevertheless, the biological basis of the adaptive mapping-based DNA base interaction needs to be further explored.

\section{Conclusion}

This paper introduces an adaptive symbol-to-number mapping into wavelet transform. An adaptive WT, namely BWT, is proposed for the analysis of a DNA sequence. Such adaptive mapping arises from the 4-channel indicator sequence structure of the DNA sequence. Besides the function of transferring from symbol to number, the adaptive mapping can adjust the weight value of each channel to maximise the energy distribution of the whole BWT output. BWT can therefore present greater energy distribution in the experiments of analysing synthetic and real DNA sequences. In conclusion, this new BWT will be useful for the detection of hidden structural patterns in future DNA sequence analysis.

\section{Acknowledgements}

This research was supported by Hong Kong Innovation and Technology Fund (ITS/114/01), Standard Telecommunications Ltd and IDT Technology Ltd.

\section{Notes}

1 In this paper, 'energy' is defined as the square of coefficient.

\section{References}

Anastassiou D. 2001. Genomic signal processing. IEEE Signal Processing Magazine, 18(4):8-20.

Arneodo A, Bacry E, Graves PV et al. 1995. Characterizing long-range correlations in DNA sequences from wavelet analysis. Phys Rev Lett, 74:3293-6.
Benson DC. 1990. Fourier methods for biosequence analysis. Nucleic Acids Res, 18:6305-10.

Berger JA, Mitra S, Carli M et al. 2002. New approaches to genome sequence analysis based on digital signal processing [online]. In: Proceedings of the IEEE Workshop on Genomic Signal Processing and Statistics (GENSIPS). 2002 Oct 12-13; Raleigh, NC, USA Accessed 17 Oct 2003. URL: http://www.gensips.gatech.edu/ proceedings/contributed/CP2-08.pdf

Bolch KM, Arce GR. 2002. Analyzing protein sequences using signal analysis techniques. In Zhang W, Shmulevich I, eds. Computational and statistical approaches to genomics. Norwell, MA: Kluwer Acad Publ. p 113-24.

Chechetkin VR, Turygin AY. 1995. Size-dependence of three-periodicity and long-range correlations in DNA sequence. Phys Lett A, 199: 75-80.

Cosic I. 1994. Macromolecular bioactivity: is it resonant interaction between macromolecules? - theory and applications. IEEE Trans Biomed Eng, 41:1101-14.

Fitch JP, Sokhansanj B. 2000. Genomic engineering: moving beyond DNA sequence to function. Proc IEEE, 88:1949-71.

Fu LM. 1999. An expert network for DNA sequence analysis. IEEE Intell Syst, 14:65-71.

Herzel H, Trifonov EN, Weiss O et al. 1998. Interpreting correlations in biosequences. Phys A, 249:449-59.

Johnson DH, Dudgeon DE. 1993. Array signal processing: concepts and techniques. Englewood Cliffs, NJ: Prentice-Hall.

Moore SK. 2000. Understanding the human genome. IEEE Spectr, 37: 33-5.

Trifonov EN. 1998. 3-, 10.5-, 200- and 400-base periodicities in genome sequences. Phys A, 249:511-16.

Veljkovic V, Cosic I, Dimitrijevic B et al. 1985. Is it possible to analyze DNA and protein sequences by the methods of digital signal processing? IEEE Trans Biomed Eng, 32:337-41.

Voss RV. 1992. Evolution of long-range fractal correlations and 1/f noise in DNA base sequences. Phys Rev Lett, 68:3805-8.

Wang W, Johnson DH. 2002. Computing linear transforms of symbolic signals. IEEE Trans Signal Process, 50:628-34.

Yao J, Zhang YT. 2001. Bionic wavelet transform: a new time-frequency method based on an auditory model. IEEE Trans Biomed Eng, 48 856-63.

Yao J, Zhang YT. 2002. The application of bionic wavelet transform to speech signal processing in cochlear implants using neural network simulations. IEEE Trans Biomed Eng, 49:1299-309.

Zhang XY, Chen F, Zhang YT et al. 2002. Signal processing techniques in genomic engineering. Proc IEEE, 90:1822-33. 

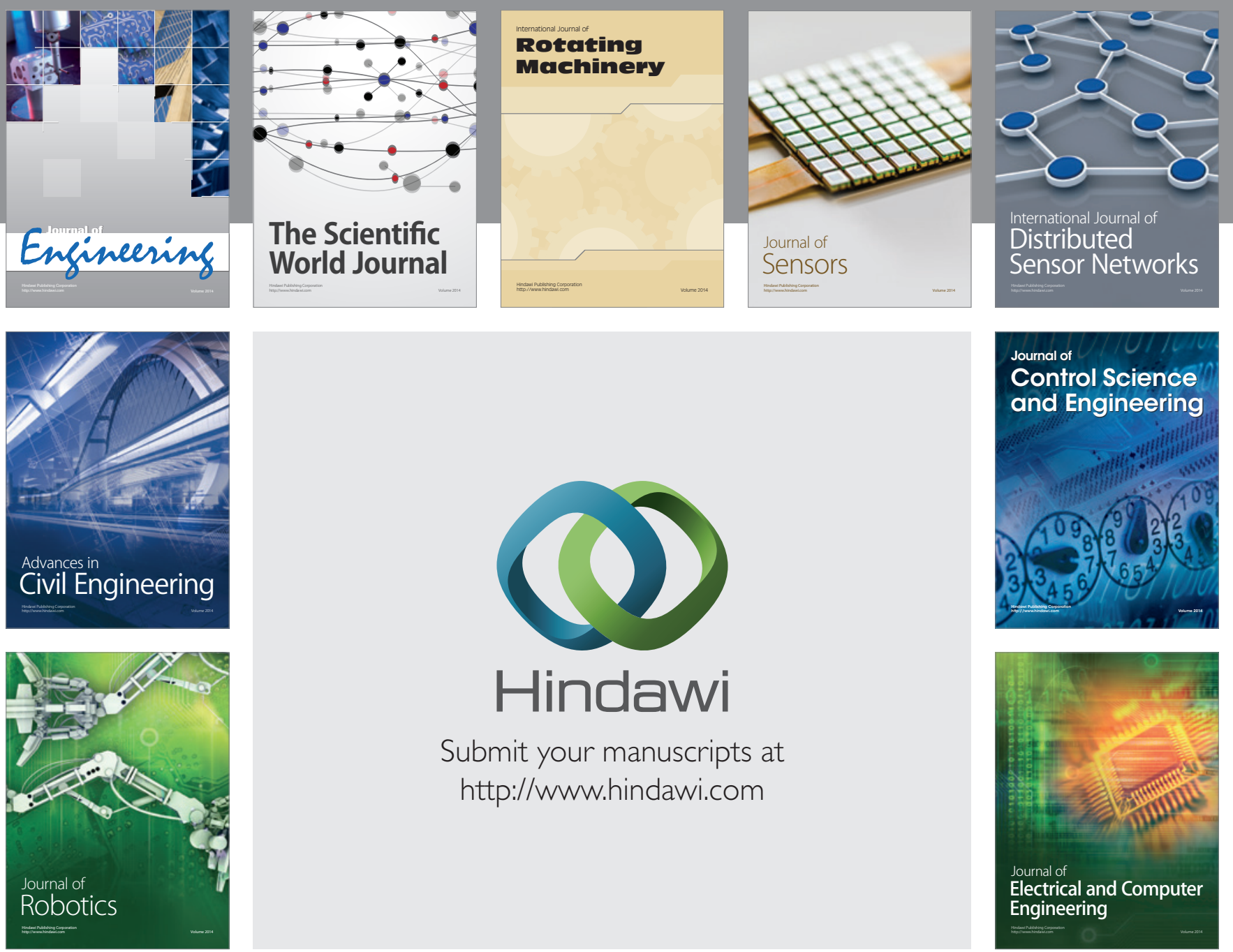

Submit your manuscripts at

http://www.hindawi.com
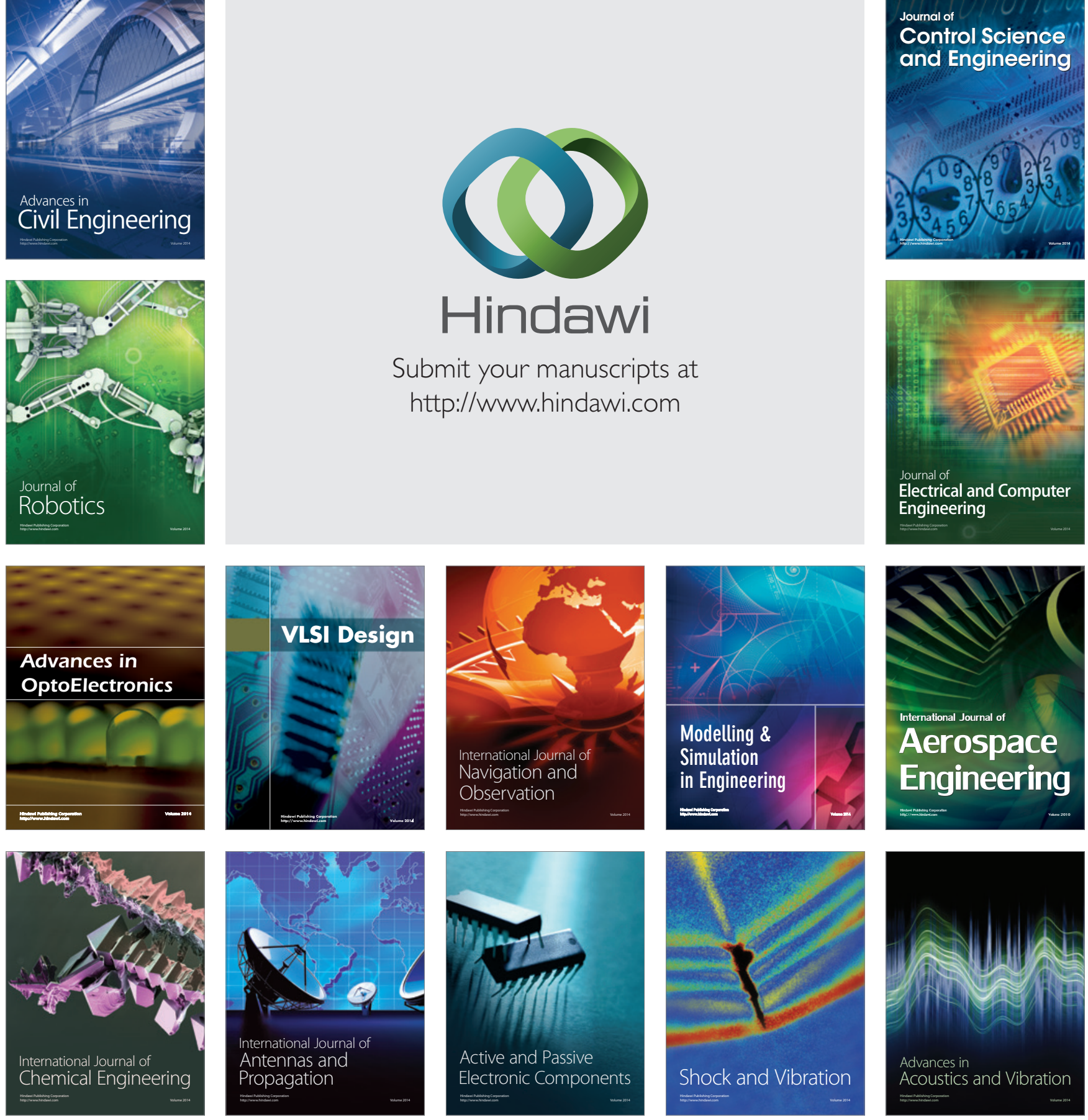